\title{
Investigation and analysis on the application of peripheral blood specimens for routine blood testing by laboratory physicians
}

\author{
Zeping Han ${ }^{1,2}$, Jinhua $\mathrm{He}^{1,2}$, Xingyi Xie ${ }^{2}$, Jianyuan Fang ${ }^{2}$, Yuguang $\mathrm{Li}^{3}$ \\ ${ }^{1}$ Central Laboratory, Guangzhou Panyu District Central Hospital, Guangzhou, China; ${ }^{2}$ Department of Laboratory Medicine, Guangzhou Panyu \\ District Central Hospital, Guangzhou, China; ${ }^{3} \mathrm{He}$ Xian Memorial Hospital, Southern Medical University, Guangzhou, China \\ Contributions: (I) Conception and design: Z Han; (II) Administrative support: Y Li; (III) Provision of study materials or patients: X Xie; (IV) \\ Collection and assembly of data: J Fang; (V) Data analysis and interpretation: Z Han, J He; (VI) Manuscript writing: All authors; (VII) Final approval \\ of manuscript: All authors. \\ Correspondence to: Yuguang Li. He Xian Memorial Hospital, Southern Medical University, Guangzhou, China. Email: lyg_py@126.com.
}

\begin{abstract}
Backgroundk Owing to its simplicity, speed, small incision, and low cost, peripheral blood collection is widely used in routine blood testing. However, there are certain differences between the detection results of peripheral and venous blood specimens. This investigation and analysis aimed to investigate and analyze the status and opinions of laboratory physicians on the application of peripheral blood for routine blood testing.

Methods: A questionnaire was used to investigate and analyze the current status of laboratory physicians with regards to routine peripheral blood testing. The content of the questionnaire included the following aspects: the proportion of peripheral blood specimens, the difficulty of detecting peripheral blood, factors affecting the results of peripheral blood specimens, the proportion of the simultaneous detection of blood test and C-reactive protein (CRP), as well as the proportion and ideals of hypersensitive CRP (hs-CRP), which included a total of 10 re-examination rules.
\end{abstract}

Results: Laboratory physicians from 410 hospitals participated in the investigation. The proportion of routine blood tests using peripheral blood specimens in the laboratory departments of tertiary hospitals was low $(\mathrm{P}=0.006)$. Difficulties in routine blood tests with peripheral blood specimens were dominated by insufficient blood volume $(67.8 \%)$. The factors affecting the results of routine blood tests with peripheral blood specimens were dominated by the mixing method $(86.6 \%)$. When abnormal results were determined by routine blood tests using peripheral blood specimens, they were retested most commonly by making slide smears $(82.4 \%)$ or re-examined using the remaining peripheral blood $(66.1 \%)$. The same rules for reexamination of peripheral and venous blood were applied in most medical institutions (86.1\%). When the platelet count decreased in routine blood tests using peripheral blood samples, the most common measures included sample agglutination checks (88.5\%), instrument alarm message checks (82.4\%), and making slide smears for re-examination (73.6\%). More laboratory physicians expected blood analyzers to be integrated instruments that could provide both routine blood and hs-CRP testing (80.5\%).

Conclusions: There are numerous difficulties in routine blood testing using peripheral blood, and thus, more convenient and accurate blood analysis instruments should be developed.

Keywords: Test; peripheral blood; routine blood; ultrasensitive C-reactive protein (ultrasensitive CRP); hematology analyzer

Submitted Jun 23, 2021. Accepted for publication Sep 03, 2021.

doi: 10.21037/apm-21-2068

View this article at: https://dx.doi.org/10.21037/apm-21-2068 


\section{Introduction}

Routine blood tests are one of the most basic and common tests in clinical practice, which could provide clinicians with the scientific basis for the diagnosis, treatment, and prognostic evaluation of many diseases (1). Therefore, routine blood tests are widely used in clinical practice, especially after the gradual improvement of medical standards, and almost every patient who requires blood detection may undergo a routine blood test. C-reactive protein (CRP) is an acute temporal reactive protein produced by liver cells; the amount of CRP synthesized is greatly increased when the body is infected by pathogens or inflammation occurs in the body (2). The hypersensitive CRP (hs-CRP) is an ultra-sensitive detection technology used in clinical laboratories, which can accurately detect low concentrations of CRP, improve the sensitivity and accuracy of the test, and detect sensitive indicators for distinguishing low levels of inflammation or infection, and often improve diagnostic accuracy in conjunction with routine blood tests (3-5). Owing to its simplicity, speed, small incision, and other advantages, peripheral blood collection is widely used in clinical practice, especially in outpatient clinics or primary care units. Furthermore, it is more popular among clinicians and patients for its lower cost and shorter testing time, as well as the indirect reduction of other unnecessary index tests (6-9).

However, a large number of studies have found some discrepancies between the results of peripheral blood testing and those of venous blood specimens $(10,11)$. The reasons for this include the following: firstly, the improper method of collecting peripheral blood specimens can easily cause specimen dilution, agglutination, or contamination; secondly, there are relatively small amounts of specimens; and thirdly, the accuracy of the testing instrument is insufficient $(12,13)$. In addition, because of the frequent need for simultaneous testing of hs-CRP, venous blood testing is applied in many medical institutions, which leads to increased trauma, prolonged time for blood specimen collection, difficulties in blood collection for some patients, and so on. To further investigate and analyze the status and opinions of laboratory physicians on the application of peripheral blood for routine blood testing, simultaneous hs-CRP testing, and others in China, we conducted a questionnaire survey, and the results are reported below.

We present the following article in accordance with the SURGE reporting checklist (available at https://dx.doi. org/10.21037/apm-21-2068).

\section{Methods}

\section{Object of the study}

In May 2020, a questionnaire survey was conducted on physicians (one physician or technologist per hospital, with junior title or above) in the laboratory departments of 410 hospitals in China.

All procedures performed in this study involving human participants were in accordance with the Declaration of Helsinki (as revised in 2013). The study was approved by Medical Ethics Committee of Guangzhou Panyu District Central Hospital (No.: PYRC-2021-054) and informed consent was taken from all the patients.

\section{Questionnaire}

A questionnaire was designed using the questionnaire star system (www.wjx.cn), which primarily included the following 10 items: the proportion of peripheral blood specimens, the difficulty of detecting peripheral blood, factors affecting the results of peripheral blood specimens, the most common methods when the routine blood test results of peripheral blood specimens are abnormal, the degree of consistency between the re-examination rules of peripheral and venous blood, treatment measures for the dropped platelet counts, proportion of the simultaneous detection of blood test and CRP, as well as the proportion and ideals of hs-CRP.

\section{Statistical analysis}

After the survey, the EXCEL (Microsoft Corporation, http://www.microsoft.com) table was exported, and the data were organized and imported into SPSS 23.0 statistical software (IBM, https://www.ibm.com) for analysis. Data in this study were all qualitative data, expressed as cases and percentages, and the comparison among groups was conducted using the chi-square test. $\mathrm{P}<0.05$ was considered statistically significant.

\section{Results}

\section{General status}

In this study, 28 provinces and 410 medical institutions in laboratory departments in China were investigated, and a total of 410 valid questionnaires were collected, with $100 \%$ recovery rate and pass rate. There were 251 tertiary 
Table 1 Accounted distribution of peripheral blood specimens for routine blood testing

\begin{tabular}{|c|c|c|c|c|}
\hline Distribution of peripheral blood & $\leq 10 \%$ & $10-20 \%$ & $20-50 \%$ & $\geq 50 \%$ \\
\hline Secondary hospital $(n=126)$ & 47 & 40 & 31 & 8 \\
\hline Primary hospital $(n=33)$ & 9 & 9 & 7 & 8 \\
\hline Total $(n=410)$ & 173 & 125 & 78 & 34 \\
\hline
\end{tabular}

hospitals (61.2\%, including 199 tertiary general hospitals and 52 tertiary specialty hospitals), 126 secondary hospitals (30.7\%, including 101 secondary general hospitals and 25 secondary specialty hospitals), and 33 medical institutions at the level of primary hospitals and below (8.0\%).

\section{Percentage of peripheral blood specimens during routine blood testing}

A total of 117 tertiary hospitals (46.6\%), 47 (37.3\%) secondary hospitals, and $9(27.3 \%)$ medical institutions at the level of primary hospitals and below had no more than $10 \%$ peripheral blood specimens during routine blood testing. It was suggested that there was a low proportion of routine blood tests using peripheral blood specimens in the laboratory department of tertiary care hospitals $(\mathrm{P}=0.006)$. A total of 34 medical institutions $(8.3 \%)$ conducted the main routine blood test with peripheral blood specimens, and all of them were specialized hospitals or primary care institutions, as shown in Table 1.

\section{Problems associated with the use of peripheral blood specimens for routine blood testing}

The following problems were associated with the use of peripheral blood specimens for routine blood testing: (I) the difficulties in routine blood testing by peripheral blood specimens were dominated by insufficient blood volume $(67.8 \%)$ and risk of contamination $(58.5 \%)$; (II) the factors affecting the results of routine blood testing with peripheral blood specimens were dominated by the mixing method (86.6\%) and placement time (76.6\%); (III) when the results of routine blood testing with peripheral blood specimens were abnormal, the most common methods included making slide smears for retesting $(82.4 \%)$ and retesting with the remaining peripheral blood (66.1\%); (IV) when peripheral blood needed slide smears to be made for testing, manually making slide smears $(83.7 \%$ ) was primarily chosen;
(V) the rules for retesting peripheral blood in most medical institutions were the same as those for retesting venous blood $(86.1 \%)$; (VI) when the platelet count in routine blood tests with peripheral blood specimens decreased, the most common measures included sample agglutination checks $(88.5 \%)$, instrument alarm message checks (82.4\%), and making slide smears for retesting $(73.6 \%)$; and (VII) the reason for the low rate of peripheral blood retesting was primarily "lack of time" (59.0\%) (Table 2).

\section{Expectations of laboratory physicians for hematology analyzers}

The percentage of testing routine blood and testing hsCRP at the same time did not differ among the different levels of hospitals $(\mathrm{P}=0.807)$. More laboratory physicians expected hematology analyzers to be integrated instruments that could provide both routine blood and hs-CRP testing (80.5\%) (Table 3).

\section{Discussion}

Routine blood and hs-CRP tests often need to be performed simultaneously, and although the different blood collection methods exert a certain influence on the results (14-16), it is within the acceptable range in most cases $(17,18)$. In this study, it was found that routine blood tests using peripheral blood specimens are widely used nationwide in primary care institutions as well as in large tertiary general hospitals, such as those in Beijing, Shanghai, Guangzhou, and Shenzhen. However, the percentage of application varies among all grades of medical institutions. This may be due to the fact that there are often relatively complex conditions among patients attending large medical institutions, who often have to undergo biochemical and immunological examinations at the same time on the basis of routine blood tests, resulting in more cases of venous blood collection. Also, there were numerous inpatients in secondary hospitals and higher- 
Table 2 Problems associated with the use of peripheral blood for routine blood testing

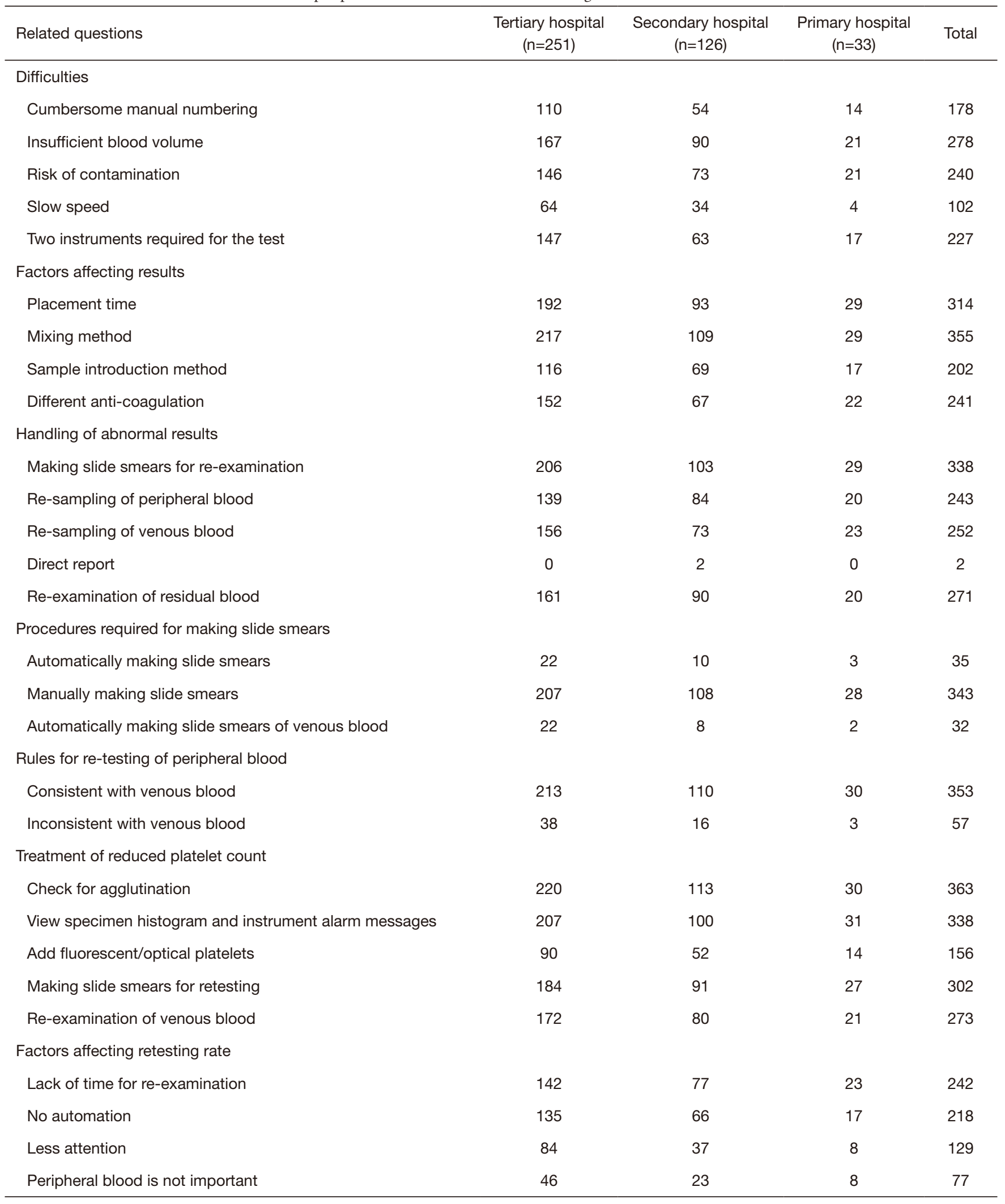


Table 3 Percentage of simultaneous routine blood test and hs-CRP tests and physician expectations of hematology analyzers

\begin{tabular}{|c|c|c|c|c|}
\hline Percentage and expectations & $\begin{array}{l}\text { Tertiary hospital } \\
\qquad(n=251)\end{array}$ & $\begin{array}{l}\text { Secondary hospital } \\
\qquad(n=126)\end{array}$ & $\begin{array}{l}\text { Primary hospital } \\
\qquad(n=33)\end{array}$ & Tota \\
\hline \multicolumn{5}{|l|}{ Percentage of simultaneous tests } \\
\hline$\leq 10-20 \%$ & 68 & 30 & 7 & 105 \\
\hline \multicolumn{5}{|l|}{ Physician expectations of hematology analyzers } \\
\hline Routine blood and hs-CRP integrated machine & 201 & 105 & 24 & 330 \\
\hline Automated batch test for venous and peripheral blood & 174 & 92 & 25 & 291 \\
\hline Fully automated and micro making slide smears & 171 & 93 & 24 & 288 \\
\hline
\end{tabular}

hs-CRP, hypersensitive C-reactive protein.

grade hospitals who underwent the relevant laboratory tests with venous blood collection mostly. Moreover, the hematology analyzers in secondary hospitals and higher-grade hospitals may be more advanced, which can simultaneously tested for hs-CRP and other indicators, and thus, venous blood collection is often required. However, numerous studies have demonstrated that peripheral blood testing for hs-CRP is equally accurate and reproducible $(19,20)$. Furthermore, based on the investigation and analysis of the problems associated with the application of peripheral blood for routine blood testing, we observed that "insufficient blood volume" and "risk of contamination" were identified as the main difficulties in routine blood testing with peripheral blood by laboratory physicians, which also led to the factors affecting the results of peripheral blood testing being the "mixing method" and "placement time". Moreover, additional instruments are required for the testing of hs-CRP in some hospitals, involving cumbersome operation procedures. Therefore, many laboratory physicians hope that routine blood and hs-CRP testing can be automated with an integrated blood analyzer to improve efficiency, which is crucial in larger general hospitals with higher volumes of outpatients, as well as in seasons with high respiratory infections, especially.

In the study, we found that if the routine blood test results of peripheral blood specimens were abnormal, there was confusion and concern about the treatment method by the testing physician in clinical practice. On the one hand, if the patient is asked to collect blood from the fingertips again for re-examination, especially when the venous blood needs to be taken for re-examination, this often leads to patient dissatisfaction and even causes medical complaints and disputes. On the other hand, the amount of peripheral blood specimens may be insufficient when the instrument is retested, and it is often chosen to make slide smears in clinical practice. Also, the quality of making slide smears directly affects the accuracy of the report. Our survey results showed that manually making slide smears $(83.7 \%)$ was primarily chosen for the actual re-examination, which could save testing time for physicians as the test results greatly depend on the experience of the operator. It is worth affirming that in the majority of medical institutions, the rules of peripheral blood re-examination are consistent with those of venous blood re-examination, which ensures the accuracy of test results to a certain extent. However, more than half of the examining physicians believe proposed "lack of time" as the reason for the lower retest rate of peripheral blood than venous blood, which is also due to the insufficient amount of peripheral blood and the fact that retesting often requires the manual making slide smears. When the platelet count of peripheral blood decreases, the examiner will take a number of steps to confirm the accuracy of the result, including checking the sample for agglutination, checking the histogram of the specimen and 
the alarm message of instrument, making slide smears for re-examination, and asking the patient to take venous blood for re-examination if necessary. In general, it is necessary to decide the method of re-examination according to the degree of platelet decline. If the decline is severe, further venous blood testing is taken to confirm the result.

Based on the above findings and the analysis of our investigation, combined with the current clinical practices and the recent respiratory disease epidemic, we believe that in order to improve the efficiency and accuracy of routine blood testing, a hematology analyzer that can simultaneously perform routine blood and hs-CRP tests should be further developed and integrated, and its automation level, testing accuracy, and batch testing capability should be improved.

\section{Acknowledgments}

Funding: None.

\section{Footnote}

Reporting Checklist: The authors have completed the SURGE reporting checklist. Available at https://dx.doi. org/10.21037/apm-21-2068

Data Sharing Statement: Available at https://dx.doi. org/10.21037/apm-21-2068

Conflicts of Interest: All authors have completed the ICMJE uniform disclosure form (available at https://dx.doi. org/10.21037/apm-21-2068). The authors have no conflicts of interest to declare.

Ethical Statement: The authors are accountable for all aspects of the work in ensuring that questions related to the accuracy or integrity of any part of the work are appropriately investigated and resolved. All procedures performed in this study involving human participants were in accordance with the Declaration of Helsinki (as revised in 2013). The study was approved by Medical Ethics Committee of Guangzhou Panyu District Central Hospital (No.: PYRC-2021-054) and informed consent was taken from all the patients.

Open Access Statement: This is an Open Access article distributed in accordance with the Creative Commons Attribution-NonCommercial-NoDerivs 4.0 International License (CC BY-NC-ND 4.0), which permits the non- commercial replication and distribution of the article with the strict proviso that no changes or edits are made and the original work is properly cited (including links to both the formal publication through the relevant DOI and the license). See: https://creativecommons.org/licenses/by-nc-nd/4.0/.

\section{References}

1. Barger AM. The complete blood cell count: a powerful diagnostic tool. Vet Clin North Am Small Anim Pract 2003;33:1207-22.

2. Sproston NR, Ashworth JJ. Role of C-reactive protein at sites of inflammation and infection. Front Immunol 2018;9:754.

3. Moutachakkir M, Lamrani Hanchi A, Baraou A, et al. Immunoanalytical characteristics of $\mathrm{C}$-reactive protein and high sensitivity C-reactive protein. Ann Biol Clin (Paris) 2017;75:225-9.

4. Chen L, Zhang J, Zhang W, et al. Correlation between C-reactive protein/albumin and contralateral hip refracture after total hip arthroplasty in elderly patients with hip fractures. Ann Palliat Med 2020;9:1055-61.

5. Oda E, Kawai R. Comparison between high-sensitivity C-reactive protein (hs-CRP) and white blood cell count (WBC) as an inflammatory component of metabolic syndrome in Japanese. Intern Med 2010;49:117-24.

6. Rooney K. Capillary blood sampling from the finger. Methods Mol Biol 2018;1735:267-72.

7. Wang Z, Lu Y, Han J. Peripheral blood microRNAs: a novel tool for diagnosing disease? Intractable Rare Dis Res 2012;1:98-102.

8. Mohammed EA, Mohamed MM, Far BH, et al. Peripheral blood smear image analysis: A comprehensive review. J Pathol Inform 2014;5:9.

9. Adewoyin AS, Nwogoh B. Peripheral blood film - a review. Ann Ib Postgrad Med 2014;12:71-9.

10. Chavan P, Bhat V, Tiwari M, et al. Comparison of complete blood count parameters between venous and capillary blood in oncology patients. J Lab Physicians 2016;8:65-6.

11. Kayiran SM, Ozbek N, Turan M, et al. Significant differences between capillary and venous complete blood counts in the neonatal period. Clin Lab Haematol 2003;25:9-16.

12. Daae LN, Halvorsen S, Mathisen PM, et al. A comparison between haematological parameters in 'capillary' and venous blood from healthy adults. Scand J Clin Lab Invest 1988;48:723-6. 
13. Yang ZW, Yang SH, Chen L, et al. Comparison of blood counts in venous, fingertip and arterial blood and their measurement variation. Clin Lab Haematol 2001;23:155-9.

14. Papa F, Rongioletti M, Ventura MD, et al. Blood cell counting in neonates: a comparison between a low volume micromethod and the standard laboratory method. Blood Transfus 2011;9:400-6.

15. Wang L, Wu L, Lang Y, et al. Association between highsensitivity C-reactive protein levels and clinical outcomes in acute ischemic stroke patients treated with endovascular therapy. Ann Transl Med 2020;8:1379.

16. Papa F, Rongioletti M, Majolini MB, et al. Fast bedside measurement of blood count and C-reactive protein in newborns compared with conventional methods. Clin Lab 2012;58:951-7.

17. Oudatzis G, Tsagarakis NJ, Paterakis G, et al. Evaluation of automated capillary complete blood counts for routine

Cite this article as: Han Z, He J, Xie X, Fang J, Li Y. Investigation and analysis on the application of peripheral blood specimens for routine blood testing by laboratory physicians. Ann Palliat Med 2021;10(9):9516-9522. doi: 10.21037/apm-212068 clinical decision making in a large cohort of hematological patients, using Mindray BC-3000 Plus Auto and Sysmex XE-5000 hematology analyzers. Int J Lab Hematol 2020;42:565-72.

18. Hollis VS, Holloway JA, Harris S, et al. Comparison of venous and capillary differential leukocyte counts using a standard hematology analyzer and a novel microfluidic impedance cytometer. PLoS One 2012;7:e43702.

19. Papaevangelou V, Papassotiriou I, Sakou I, et al. Evaluation of a quick test for $\mathrm{C}$-reactive protein in a pediatric emergency department. Scand J Clin Lab Invest 2006;66:717-21.

20. Ivaska L, Niemelä J, Leino P, et al. Accuracy and feasibility of point-of-care white blood cell count and C-reactive protein measurements at the pediatric emergency department. PLoS One 2015;10:e0129920.

(English Language Editor: A. Kassem) 